Proceedings of the Edinburgh Mathematical Society (2006) 49, 87-100 (C)

DOI:10.1017/S0013091504000938 Printed in the United Kingdom

\title{
LOW-REGULARITY SOLUTIONS FOR THE OSTROVSKY EQUATION
}

\author{
ZHAOHUI HUO ${ }^{1}$ AND YUELING JIA ${ }^{2}$ \\ ${ }^{1}$ Department of Mathematics, School of Sciences, \\ Beijing University of Aeronautics and Astronautics, Beijing 100083, \\ People's Republic of China (huozhaohui@yahoo.com.cn) \\ ${ }^{2}$ National Key Laboratory of Computational Physics, Institute of Applied Physics \\ and Computational Mathematics, PO Box 8009, Beijing 100088, \\ People's Republic of China (jiayueling@yahoo.com.cn)
}

(Received 20 September 2004)

Abstract The well-posedness of the Ostrovsky equation is considered. Local well-posedness for data in $\tilde{H}^{s}(\mathbb{R})\left(s \geqslant-\frac{1}{8}\right)$ and global well-posedness for data in $\tilde{L}^{2}(\mathbb{R})$ are obtained.

Keywords: Ostrovsky equation; Fourier restriction norm; Cauchy problem; low-regularity solutions

2000 Mathematics subject classification: Primary 35A07; 35D10; 35G25; 35Q53

\section{Introduction}

Our aim is to study low-regularity solutions to the Cauchy problem of the Ostrovsky equation:

$$
\begin{gathered}
\left(u_{t}-\beta u_{x x x}+\left(u^{2}\right)_{x}\right)_{x}=\gamma u, \quad(x, t) \in \mathbb{R} \times \mathbb{R}, \\
u(x, 0)=u_{0}(x) \in \tilde{H}^{s}(\mathbb{R}),
\end{gathered}
$$

where $\beta$ and $\gamma$ are real constants and $\beta \gamma \neq 0$. The space $\tilde{H}^{s}$ is defined by

$$
\tilde{H}^{s}=\left\{f \in H^{s}(\mathbb{R}): \mathcal{F}_{x}^{-1}\left(\frac{\hat{f}(\xi)}{\xi}\right) \in H^{s}(\mathbb{R})\right\}
$$

with the norm

$$
\|f\|_{\tilde{H}^{s}}=\|f\|_{H^{s}}+\left\|\mathcal{F}_{x}^{-1}\left(\frac{\hat{f}(\xi)}{\xi}\right)\right\|_{H^{s}} .
$$

Integrating Equation (1.1) with respect to $x$ over $\mathbb{R}$ for $\gamma \neq 0$, we obtain that

$$
\int u(x, t) \mathrm{d} x=0 .
$$

That means that any local solution of (1.1) satisfies the above identity. 
The Ostrovsky equation $[\mathbf{1 0}]$ governs the propagation of weakly nonlinear long surface and internal waves of small amplitude in a rotating fluid. The liquid is assumed to be incompressible and inviscid. After suitable scaling, the equation can be written as above [6]. Parameter $\beta$ determines the type of dispersion:

(i) $\beta=-1$ (negative dispersion) in the cases of surface and internal waves in the ocean and surface waves in a shallow channel with an uneven bottom; and

(ii) $\beta=1$ (positive dispersion) in the cases of capillary waves on the surface of a liquid or oblique magneto-acoustic waves in plasma $[\mathbf{1}, \mathbf{5}, \mathbf{6}]$.

Equation (1.1) models the situation when nonlinearity, dispersion and rotation are taken into account but dissipation is ignored.

Denote the homogeneous derivative of order $s$ with respect to variable $x$ by $D_{x}^{s}$. Rewrite Equation (1.1) in the following way:

$$
u_{t}-\beta u_{x x x}+\left(u^{2}\right)_{x}=\gamma D_{x}^{-1} u .
$$

Setting $\gamma=0$ in (1.3) we can obtain the Korteweg-de Vries (KdV) equation

$$
u_{t}-\beta u_{x x x}+\left(u^{2}\right)_{x}=0 .
$$

Regarding the low-regularity solution of the KdV equation, Kenig et al. [9] found that the Cauchy problem of the KdV equation is locally well-posed for data in $H^{s}\left(s>-\frac{3}{4}\right)$ by using the so-called Fourier restriction norm (Bourgain function spaces).

The Fourier restriction norm method was first introduced by Bourgain $[\mathbf{3}, \mathbf{4}]$ to study the KdV and nonlinear Schrödinger equations in the periodic case. It was simplified by Kenig et al. $[\mathbf{8}, \mathbf{9}]$.

Recently, Varlamov and Liu [13] found that the problem (1.1), (1.2) is locally wellposed in the space $\tilde{H}^{s}\left(s>\frac{3}{2}\right)$ with the condition $\gamma>0$ by using the method of parabolic regularization.

In this paper, we consider the well-posedness of problem (1.1), (1.2) in $\tilde{H}^{s}\left(s \geqslant-\frac{1}{8}\right)$ without the restriction $\gamma>0$ (here the condition $\gamma>0$ is also redundant). We obtain our results using the Fourier restriction norm method.

Therefore, we use the integral equivalent formulation of the problem

$$
u(x, t)=S(t) u_{0}-\int_{0}^{t} S\left(t-t^{\prime}\right) \partial_{x}\left(u^{2}\right)\left(t^{\prime}\right) \mathrm{d} t^{\prime},
$$

where

$$
S(t)=\mathcal{F}_{x}^{-1} \mathrm{e}^{-\mathrm{i} t\left(\beta \xi^{3}+(\gamma / \xi)\right)} \mathcal{F}_{x}
$$

is the unitary operator associated with the linear equation. For simplicity, denote the phase function by $\phi(\xi)=\beta \xi^{3}+(\gamma / \xi)$.

One finds that the function $\phi(\xi)$ has a singularity at $\xi=0$ and non-zero points where $\phi^{\prime}(\xi)=0$ and $\phi^{\prime \prime}(\xi)=0$, which is in contrast to the phase functions of the semigroup 
of the linear KdV equation and makes the problem much more difficult. Therefore, we need to use Fourier restriction operators

$$
P^{N} f=\int_{|\xi| \geqslant N} \mathrm{e}^{\mathrm{i} x \xi} \hat{f}(\xi) \mathrm{d} \xi, \quad P_{(\varepsilon, N)} f=\int_{\varepsilon \leqslant|\xi| \leqslant N} \mathrm{e}^{\mathrm{i} x \xi} \hat{f}(\xi) \mathrm{d} \xi, \quad \forall N \geqslant \varepsilon>0,
$$

to eliminate the singularity.

Moreover, the operators will be used to decompose nonlinear term $\partial_{x}\left(u^{2}\right)$. To deal with the term, we first decompose it as the high-frequency part and the corresponding low-frequency one as follows:

$$
\partial_{x}\left(u^{2}\right)=P^{N}\left\{\partial_{x}\left(u^{2}\right)\right\}+P_{(0, N)}\left\{\partial_{x}\left(u^{2}\right)\right\} .
$$

Next, we continue to decompose each term on the right-hand side of (1.7) as the summation of those products which consist of each factor acted on by the Fourier restriction operators $P^{N}$ or $P_{N}$. We will estimate each resulting term with different methods to overcome the obstacles.

Definition 1.1. For $s, b \in \mathbb{R}$, the space $X_{s, b}$ is the completion of the Schwartz function space on $\mathbb{R}^{2}$ with respect to the norm

$$
\|u\|_{X_{s, b}}=\|S(-t) u\|_{H_{x}^{s} H_{t}^{b}}=\left\|\langle\xi\rangle^{s}\langle\tau+\phi(\xi)\rangle^{b} \mathcal{F} u\right\|_{L_{\xi}^{2} L_{\tau}^{2}}
$$

where $\langle\cdot\rangle=(1+|\cdot|)$.

We define the modified Bourgain function space $\tilde{X}_{s, b}$ in a similar way to the definition of $\tilde{H}^{s}$ :

$$
\begin{aligned}
\|u\|_{\tilde{X}_{s, b}} & =\left\|\langle\xi\rangle^{s}\langle\tau+\phi(\xi)\rangle^{b} \mathcal{F} u\right\|_{L_{\xi}^{2} L_{\tau}^{2}}+\left\|\langle\xi\rangle^{s}|\xi|^{-1}\langle\tau+\phi(\xi)\rangle^{b} \mathcal{F} u\right\|_{L_{\xi}^{2} L_{\tau}^{2}} \\
& =\|u\|_{X_{s, b}}+\left\|D_{x}^{-1} u\right\|_{X_{s, b}} .
\end{aligned}
$$

In our arguments, we will use the trivial embedding relation

$$
\|u\|_{\tilde{X}_{s_{1}, b_{1}}} \leqslant\|u\|_{\tilde{X}_{s_{2}, b_{2}}}
$$

whenever $s_{1} \leqslant s_{2}, b_{1} \leqslant b_{2}$.

Let $\psi \in C_{0}^{\infty}(\mathbb{R})$ with $\psi=1$ on $\left[-\frac{1}{2}, \frac{1}{2}\right]$ and $\operatorname{supp} \psi \subset[-1,1]$. Define $\psi_{\delta}(\cdot)=\psi\left(\delta^{-1}(\cdot)\right)$ for some $\delta \in \mathbb{R}$.

Define $A \sim B$ using the statement $A \leqslant C_{1} B$ and $B \leqslant C_{1} A$ for some constant $C_{1}>0$, and define $A \ll B$ through the statement $A \leqslant\left(1 / C_{2}\right) B$ for some large enough constant $C_{2}>0$.

Denote $\hat{u}(\tau, \xi)=\mathcal{F} u$ by the Fourier transform of $u$ in both variables $t$ and $x$, and $\mathcal{F}_{(\cdot)} u$ by the Fourier transform of $u$ only in the (.) variable.

Let us introduce some variables:

$$
\sigma=\tau+\beta \xi^{3}+\frac{\gamma}{\xi}, \quad \sigma_{1}=\tau_{1}+\beta \xi_{1}^{3}+\frac{\gamma}{\xi_{1}}, \quad \sigma_{2}=\tau_{2}+\beta \xi_{2}^{3}+\frac{\gamma}{\xi_{2}} .
$$


From the above variables, it is easy to obtain

$$
\sigma-\sigma_{1}-\sigma_{2}=3 \beta \xi \xi_{1} \xi_{2}\left(1-\gamma \frac{\xi_{1}^{2}+\xi_{2}^{2}+\xi_{1} \xi_{2}}{3 \beta\left(\xi \xi_{1} \xi_{2}\right)^{2}}\right)
$$

Throughout this paper, we define $\int_{\star} \mathrm{d} \delta$, the convolution integral, to be

$$
\int_{\xi=\xi_{1}+\xi_{2} ; \tau=\tau_{1}+\tau_{2}} \mathrm{~d} \tau_{1} \mathrm{~d} \tau_{2} \mathrm{~d} \xi_{1} \mathrm{~d} \xi_{2}
$$

Next, we will give some useful notation for multilinear expressions [12]. Let $Z$ be any abelian additive group with an invariant measure $\mathrm{d} \xi$. For any integer $k \geqslant 2$, we define $\Gamma_{k}(Z)$ to be the 'hyperplane'

$$
\Gamma_{k}(Z)=\left\{\left(\xi_{1}, \ldots, \xi_{k}\right) \in Z^{k}: \xi_{1}+\cdots+\xi_{k}=0\right\},
$$

and define a $[k ; Z]$-multiplier to be any function $m: \Gamma_{k}(Z) \rightarrow \mathbb{C}$.

If $m$ is a $[k ; Z]$-multiplier, then we define $\|m\|_{[k ; Z]}$ to be the best constant, such that the inequality

$$
\left|\int_{\Gamma_{k}(Z)} m(\xi) \prod_{j=1}^{k} f_{j}\left(\xi_{j}\right)\right| \leqslant\|m\|_{[k ; Z]} \prod_{j=1}^{k}\left\|f_{j}\right\|_{L_{2}(Z)}
$$

holds for all test functions $f_{j}$ defined on $Z$.

It is clear that $\|m\|_{[k ; Z]}$ determines a norm on $m$ for test functions at least; we are interested in obtaining the good boundedness on the norm. In this paper, we let $Z=$ $\mathbb{R} \times \mathbb{R}$

We now give the statement of the main results.

Theorem 1.2. Let $s \geqslant-\frac{1}{8}, b$ be close enough to $\frac{1}{2}$ satisfying $b>\frac{1}{2}$. Then there exists some constant $T>0$, and problem (1.1), (1.2) admits a unique local solution $u(x, t) \in C\left([0, T] ; \tilde{H}^{s}\right) \cap \tilde{X}_{s, b}$ with $u_{0} \in \tilde{H}^{s}$. Moreover, given $t \in(0, T)$, the mapping $u_{0} \rightarrow u$ is Lipschitz continuous from $\tilde{H}^{s}$ to $C\left([0, T] ; \tilde{H}^{s}\right)$.

Multiplying (1.3) by $u$ and integrating it over $\mathbb{R}$, we deduce that

$$
\frac{1}{2} \frac{\mathrm{d}}{\mathrm{d} t}\left(\int|u(t, x)|^{2} \mathrm{~d} x\right)=\int\left(\frac{1}{2} \gamma\left(\left(D_{x}^{-1} u\right)^{2}\right)_{x}-\frac{1}{2} \beta\left(\left(u_{x}\right)^{2}\right)_{x}-\frac{2}{3}\left(u^{3}\right)_{x}\right) \mathrm{d} x=0 .
$$

Hence it follows the law of conservation momentum:

$$
\int|u(t, x)|^{2} \mathrm{~d} x=\text { const. }
$$

We then have global well-posedness of problem (1.1), (1.2) for data in $\tilde{L}^{2}$.

Theorem 1.3. For $s=0$, the solution obtained in Theorem 1.2 can be extended to a global one. 


\section{Preliminary estimates}

In this section, several estimates will be deduced. We will use the following notation:

$$
\begin{gathered}
\|f\|_{L_{x}^{p} L_{t}^{q}}=\left(\int_{-\infty}^{\infty}\left(\int_{-\infty}^{\infty}|f(x, t)|^{q} \mathrm{~d} t\right)^{p / q} \mathrm{~d} x\right)^{1 / p}, \\
\|f\|_{L_{t}^{q} L_{x}^{p}}=\left(\int_{-\infty}^{\infty}\left(\int_{-\infty}^{\infty}|f(x, t)|^{p} \mathrm{~d} x\right)^{q / p} \mathrm{~d} t\right)^{1 / q}, \\
\|f\|_{L_{t}^{\infty} H_{x}^{s}}=\|\| f\left\|_{H_{x}^{s}}\right\|_{L_{t}^{\infty}}, \quad \mathcal{F} F_{\rho}(\xi, \tau)=\frac{f(\xi, \tau)}{\left(1+\left|\tau+\beta \xi^{3}+(\gamma / \xi)\right|\right)^{\rho}}, \\
a=\max \left(1, \sqrt[4]{\left|\frac{6 \gamma}{7 \beta}\right|}, \sqrt[2]{\left|\frac{\gamma}{3 \beta}\right|} .\right.
\end{gathered}
$$

Lemma 2.1. The group $\{S(t)\}_{-\infty}^{+\infty}$ satisfies

$$
\begin{aligned}
\left\|D_{x} P^{a} S(t) u_{0}\right\|_{L_{x}^{\infty} L_{t}^{2}} & \leqslant C\left\|u_{0}\right\|_{L^{2}}, \\
\left\|D_{x}^{-1 / 4} P^{a} S(t) u_{0}\right\|_{L_{x}^{4} L_{t}^{\infty}} & \leqslant C\left\|u_{0}\right\|_{L^{2}}, \\
\left\|D_{x}^{1 / 6} P^{a} S(t) u_{0}\right\|_{L_{x}^{6} L_{t}^{6}} & \leqslant C\left\|u_{0}\right\|_{L^{2}},
\end{aligned}
$$

where the constant $C$ depends on $\gamma$ and $\beta$.

Proof. First we prove (2.1). It follows that

$$
\begin{aligned}
\phi(\xi) & =\beta \xi^{3}+\frac{\gamma}{\xi}, \\
\phi^{\prime}(\xi) & =3 \beta \xi^{2}-\frac{\gamma}{\xi^{2}} \quad(\xi \neq 0), \\
\phi^{\prime \prime}(\xi) & =6 \beta \xi+2 \frac{\gamma}{\xi^{3}} \quad(\xi \neq 0) .
\end{aligned}
$$

If $|\xi| \geqslant a$, then $\phi$ is invertible, and we have

$$
\begin{aligned}
P^{a} S(t) u_{0} & =\int_{|\xi| \geqslant a} \mathrm{e}^{\mathrm{i} x \xi} \mathrm{e}^{-\mathrm{i} t \phi(\xi)} \hat{u}_{0}(\xi) \mathrm{d} \xi \\
& =\int_{\left|\phi^{-1}\right| \geqslant a} \mathrm{e}^{\mathrm{i} x \phi^{-1}} \mathrm{e}^{-\mathrm{i} t \phi} \hat{u}_{0}\left(\phi^{-1}\right) \frac{1}{\phi^{\prime}} \mathrm{d} \phi \\
& =\mathcal{F}_{t}\left(\mathrm{e}^{\mathrm{i} x \phi^{-1}} \chi_{\left\{\left|\phi^{-1}\right| \geqslant a\right\}} \hat{u}_{0}\left(\phi^{-1}\right) \frac{1}{\phi^{\prime}}\right) .
\end{aligned}
$$

Therefore, using the change of variable $\xi=\phi^{-1}$, we have

$$
\begin{aligned}
\left\|P^{a} S(t) u_{0}\right\|_{L^{2}}^{2} & =\left\|\mathcal{F}_{t}\left(\mathrm{e}^{\mathrm{i} x \phi^{-1}} \chi_{\left\{\left|\phi^{-1}\right| \geqslant a\right\}} \hat{u}_{0}\left(\phi^{-1}\right) \frac{1}{\phi^{\prime}}\right)\right\|_{L^{2}}^{2} \\
& =\int_{\left|\phi^{-1}\right| \geqslant a}\left|\hat{u}_{0}\left(\phi^{-1}\right)\right|^{2} \frac{1}{\left|\phi^{\prime}\right|^{2}} \mathrm{~d} \phi \\
& =\int_{|\xi| \geqslant a}\left|\hat{u}_{0}(\xi)\right|^{2} \frac{1}{\left|\phi^{\prime}(\xi)\right|^{2}} \phi^{\prime}(\xi) \mathrm{d} \xi
\end{aligned}
$$




$$
\begin{aligned}
& \leqslant \int_{|\xi| \geqslant a}\left|\hat{u}_{0}(\xi)\right|^{2} \frac{1}{\left|\phi^{\prime}\right|} \mathrm{d} \xi \\
& \leqslant C\left\|u_{0}\right\|_{\dot{H}^{-1}}^{2} .
\end{aligned}
$$

This implies the estimate (2.1).

Let us turn to the proof of (2.2) next. The first inequality below holds with the help of Theorem 2.5 in [7]. It can be shown that

$$
\begin{aligned}
\left\|P^{a} S(t) u_{0}\right\|_{L_{x}^{4} L_{t}^{\infty}}^{2} & \leqslant C \int_{|\xi| \geqslant a}\left|\hat{u}_{0}(\xi)\right|^{2}\left|\frac{\phi^{\prime}(\xi)}{\phi^{\prime \prime}(\xi)}\right|^{1 / 2} \mathrm{~d} \xi \\
& \leqslant C \int_{|\xi| \geqslant a}\left|\hat{u}_{0}(\xi)\right|^{2}|\xi|^{1 / 2} \mathrm{~d} \xi \\
& \leqslant C\left\|u_{0}\right\|_{H^{1 / 4}}^{2} .
\end{aligned}
$$

Therefore, we can obtain the estimate (2.2). Finally, (2.3) follows by interpolation between (2.1) and (2.2). We refer to [2] for more details of interpolation theorems.

Lemma 2.2 (see [13]). The group $\{S(t)\}_{-\infty}^{+\infty}$ satisfies

$$
\left\|S(t) u_{0}\right\|_{L_{x}^{6} L_{t}^{6}} \leqslant C\left\|u_{0}\right\|_{L^{2}}
$$

where the constant $C$ depends on $\gamma$ and $\beta$.

Remark 2.3. We can use the Vau der Corupt lemma [11] to obtain the result.

Lemma 2.4. If $\rho>\frac{1}{2}, \forall N \geqslant \varepsilon>0$, then

$$
\left\|P_{(\varepsilon, N)} F_{\rho}\right\|_{L_{x}^{2} L_{t}^{\infty}} \leqslant C\|f\|_{L_{\xi}^{2} L_{\tau}^{2}}
$$

where the constant $C$ depends on $N$ and $\varepsilon$.

Proof. The proof is similar to that of Lemma 2.2 in [8]; the details are omitted here.

Lemma 2.5. If $\rho>\frac{3}{8}$, then

$$
\left\|D_{x}^{1 / 8} P^{a} F_{\rho}\right\|_{L_{x}^{4} L_{t}^{4}} \leqslant C\|f\|_{L_{\xi}^{2} L_{\tau}^{2}}
$$

where the constant $C$ depends on $\beta$ and $\gamma$.

Proof. Using the change of variable $\tau=\lambda-\phi(\xi)$, we have

$$
\begin{aligned}
F_{\rho}(x, t) & =\int_{-\infty}^{\infty} \int_{-\infty}^{\infty} \mathrm{e}^{\mathrm{i}(x \xi+t \tau)} \frac{f(\xi, \tau)}{(1+|\tau+\phi(\xi)|)^{\rho}} \mathrm{d} \xi \mathrm{d} \tau \\
& =\int_{-\infty}^{\infty} \mathrm{e}^{\mathrm{i} t \lambda}\left(\int_{-\infty}^{\infty} \mathrm{e}^{\mathrm{i}(x \xi+t \phi(\xi))} f(\xi, \lambda+\phi(\xi)) \mathrm{d} \xi\right) \frac{\mathrm{d} \lambda}{(1+|\lambda|)^{\rho}}
\end{aligned}
$$


Therefore, by (2.3), Minkowski's integral inequality and taking $\rho>\frac{1}{2}$, one can show that

$$
\left\|D_{x}^{1 / 6} P^{a} F_{\rho}\right\|_{L_{x}^{6} L_{t}^{6}} \leqslant C \int_{-\infty}^{+\infty}\|f(\xi, \lambda+\phi(\xi))\|_{L_{\xi}^{2}} \frac{\mathrm{d} \lambda}{(1+|\lambda|)^{\rho}} \leqslant C\|f\|_{L_{\xi}^{2} L_{\tau}^{2}} .
$$

In fact, we have

$$
\left\|F_{0}\right\|_{L_{x}^{2} L_{t}^{2}} \leqslant C\|f\|_{L_{\xi}^{2} L_{\tau}^{2}}
$$

Then (2.6) follows by interpolation between (2.7) and (2.8).

Lemma 2.6. If $\rho>\frac{1}{2} \theta$ with $\theta \in[0,1]$, then

$$
\left\|D_{x}^{\theta} P^{a} F_{\rho}\right\|_{L_{x}^{2 /(1-\theta)} L_{t}^{2}} \leqslant C\|f\|_{L_{\xi}^{2} L_{\tau}^{2}}
$$

where the constant $C$ depends on $\beta$ and $\gamma$.

Proof. From the argument (2.7) and the inequality (2.1), we have, for $\rho>\frac{1}{2}$,

$$
\left\|D_{x} P^{a} F_{\rho}\right\|_{L_{x}^{\infty} L_{t}^{2}} \leqslant C\|f\|_{L_{\xi}^{2} L_{\tau}^{2}}
$$

Then (2.9) follows by interpolation between (2.8) and (2.10).

Lemma 2.7. If

$$
\rho>\frac{1}{2} \frac{3(q-2)}{2 q}
$$

then, for $2 \leqslant q \leqslant 6$, the following inequality holds:

$$
\left\|F_{\rho}\right\|_{L_{x}^{q} L_{t}^{q}} \leqslant C\|f\|_{L_{\xi}^{2} L_{\tau}^{2}}
$$

where the constant $C$ depends on $\beta$ and $\gamma$.

Proof. From the argument (2.7) and the inequality (2.4), we have, for $\rho>\frac{1}{2}$,

$$
\left\|F_{\rho}\right\|_{L_{x}^{6} L_{t}^{6}} \leqslant C\|f\|_{L_{\xi}^{2} L_{\tau}^{2}} .
$$

Then (2.11) follows by interpolation between (2.8) and (2.12).

Lemma 2.8. Assume that $f, f_{1}$ and $f_{2}$ belong to Schwartz space on $\mathbb{R}^{2}$, then

$$
\int_{\star} \overline{\hat{f}}(\xi, \tau) \hat{f}_{1}\left(\xi_{1}, \tau_{1}\right) \hat{f}_{2}\left(\xi_{2}, \tau_{2}\right) \mathrm{d} \delta=\int \bar{f} f_{1} f_{2}(x, t) \mathrm{d} x \mathrm{~d} t
$$

Proof. For simplicity, we only discuss the case of one variable:

$$
\begin{aligned}
\int_{\xi=\xi_{1}+\xi_{2}} \overline{\hat{f}}(\xi) \hat{f}_{1}\left(\xi_{1}\right) \hat{f}_{2}\left(\xi_{2}\right) \mathrm{d} \delta & =\int_{\xi=\xi_{1}+\xi_{2}} \hat{\hat{f}}(-\xi) \hat{f}_{1}\left(\xi_{1}\right) \hat{f}_{2}\left(\xi_{2}\right) \mathrm{d} \delta \\
& =\int_{\xi_{1}} \int_{\xi_{2}^{\prime}} \int_{\xi_{3}^{\prime}} \hat{\bar{f}}\left(-\xi_{3}^{\prime}\right) \hat{f}_{1}\left(\xi_{1}\right) \hat{f}_{2}\left(\xi_{2}^{\prime}-\xi_{1}\right) \mathrm{d} \xi_{1} \mathrm{~d} \xi_{2}^{\prime} \\
& =\hat{\bar{f}} * \hat{f}_{1} * \hat{f}_{2}(0)=\mathcal{F} \bar{f} f_{1} f_{2}(0) \\
& =\int \bar{f} f_{1} f_{2}(x) \mathrm{d} x
\end{aligned}
$$


Lemma 2.9 (comparison principle [12]). If $m$ and $M$ are $[k ; Z]$ multipliers and satisfy $|m(\xi)| \leqslant|M(\xi)|$ for all $\xi \in \Gamma_{k}(Z)$, then $\|m\|_{[k ; Z]} \leqslant\|M\|_{[k ; Z]}$.

\section{Linear estimates}

Lemma 3.1 (see $[8,9]$ ). If $s \in \mathbb{R}$ and $\frac{1}{2}<b<1$, then, for $u_{0} \in \tilde{H}^{s}$, we have

$$
\left\|\psi(t) S(t) u_{0}\right\|_{\tilde{X}_{s, b}} \leqslant C\left\|u_{0}\right\|_{\tilde{H}^{s}} .
$$

Lemma 3.2 (see $[8,9]$ ). If $s \in \mathbb{R}, \frac{1}{2}<b<1$ and $0<\delta \leqslant 1$, then we have

$$
\begin{aligned}
& \left\|\psi_{\delta}(t) \int_{0}^{t} S\left(t-t^{\prime}\right) f\left(t^{\prime}\right) \mathrm{d} t^{\prime}\right\|_{\tilde{X}_{s, b}} \leqslant C \delta^{b^{\prime}-b}\|f\|_{\tilde{X}_{s, b-1}}, \\
& \left\|\psi_{\delta}(t) \int_{0}^{t} S\left(t-t^{\prime}\right) f\left(t^{\prime}\right) \mathrm{d} t^{\prime}\right\|_{\tilde{L}^{2}} \leqslant C \delta^{b^{\prime}-b}\|f\|_{\tilde{X}_{s, b-1}}, \\
& \left\|\psi_{\delta}(t) F\right\|_{\tilde{X}_{s, b}} \leqslant C \delta^{1 / 2-b}\|F\|_{\tilde{X}_{s, b}} .
\end{aligned}
$$

Lemma 3.3. If $s \in \mathbb{R}, \frac{1}{2}<b<b^{\prime}<1$ and $0<\delta \leqslant 1$, then we have

$$
\left\|\psi_{\delta}(t) F\right\|_{\tilde{X}_{s, b-1}} \leqslant C \delta^{b^{\prime}-b}\|F\|_{\tilde{X}_{s, b^{\prime}-1}} .
$$

Proof. For simplicity, let $c=1-b, d=1-b^{\prime}$, then $0<d<c<\frac{1}{2}$ from the assumption. It suffices to show that

$$
\left\|\psi_{\delta} F\right\|_{H_{t}^{-c}} \leqslant C \delta^{c-d}\|F\|_{H_{t}^{-d}} .
$$

By duality, it suffices to show that

$$
\left\|\psi_{\delta} g\right\|_{H_{t}^{d}} \leqslant \delta^{c-d}\|g\|_{H_{t}^{c}}, \quad \forall g \in H_{t}^{c}
$$

First, we easily obtain

$$
\left\|\psi_{\delta} g\right\|_{L_{t}^{2}} \leqslant C \delta^{c}\|g\|_{H_{t}^{c}} .
$$

Next, by interpolation and by using the Hölder and Sobolev inequalities, we prove that

$$
\left\|\psi_{\delta} g\right\|_{\dot{H}_{t}^{d}} \leqslant C\left\|\psi_{\delta} g\right\|_{\dot{H}_{t}^{c}}^{1-(c-d) / c}\left\|\psi_{\delta} g\right\|_{L_{t}^{2}}^{(c-d) / c} \leqslant C \delta^{c-d}\left\|\psi_{\delta} g\right\|_{\dot{H}_{t}^{c}}^{1-(c-d) / c}\|g\|_{H_{t}^{c}}^{(c-d) / c} .
$$

Moreover, for $\left\|\psi_{\delta} g\right\|_{\dot{H}_{t}^{c}}$, we have

$$
\left\|\psi_{\delta} g\right\|_{\dot{H}_{t}^{c}} \leqslant C\left(\left\|\psi_{\delta}\right\|_{L_{t}^{\infty}}\|g\|_{H_{t}^{c}}+\left\|\left(D^{c} \psi_{\delta}\right) g\right\|_{L_{t}^{2}}\right) .
$$

For $\left\|\left(D^{c} \psi_{\delta}\right) g\right\|_{L_{t}^{2}}$, by the Hölder and Sobolev inequalities, we have

$$
\left\|\left(D^{c} \psi_{\delta}\right) g\right\|_{L_{t}^{2}} \leqslant\left\|D^{c} \psi_{\delta}\right\|_{L^{1 / c}}\|g\|_{H_{t}^{c}} .
$$

For $\left\|D^{c} \psi_{\delta}\right\|_{L^{1 / c}}$, by the Hölder inequality $\left(c=\frac{1}{2}-(b-c)+b-\frac{1}{2}\right.$, for some $\left.b>\frac{1}{2}\right)$, we have

$$
\left\|D^{c} \psi_{\delta}\right\|_{L^{1 / c}} \leqslant C \delta^{b-1 / 2}\left\|D^{c} \psi_{\delta}\right\|_{L^{1 /(1 / 2-(b-c))}} \leqslant C .
$$

This completes the proof. 


\section{The bilinear estimate}

One can obtain local well-posedness of problem (1.1), (1.2) by the Picard iteration method provided that

$$
\left\|\partial_{x}\left(u_{1} u_{2}\right)\right\|_{\tilde{X}_{s, b-1}} \leqslant C\left\|u_{1}\right\|_{\tilde{X}_{s, b}}\left\|u_{2}\right\|_{\tilde{X}_{s, b}}
$$

holds for some $b>\frac{1}{2}$. We only need to prove the bilinear estimate above and state the theorem as follows.

Theorem 4.1. Let $b$ be close enough to $\frac{1}{2}$ satisfying $b>\frac{1}{2}$. For $\frac{1}{2}<b^{\prime}$ and $s \geqslant-\frac{1}{8}$, we have

$$
\left\|\partial_{x}\left(u_{1} u_{2}\right)\right\|_{\tilde{X}_{s, b-1}} \leqslant C\left\|u_{1}\right\|_{\tilde{X}_{s, b^{\prime}}}\left\|u_{2}\right\|_{\tilde{X}_{s, b^{\prime}}},
$$

where the constant $C$ depends on $\beta$ and $\gamma$.

Remark 4.2. Here, the condition $s \geqslant-\frac{1}{8}$ is required only because of the mathematical technique.

Proof. By the definition of $\tilde{X}_{s, b}$, we have

$$
\left\|\partial_{x}\left(u_{1} u_{2}\right)\right\|_{\tilde{X}_{s, b-1}}=\left\|\partial_{x}\left(u_{1} u_{2}\right)\right\|_{X_{s, b-1}}+\left\|D_{x}^{-1} \partial_{x}\left(u_{1} u_{2}\right)\right\|_{X_{s, b-1}} .
$$

We only prove the bilinear estimate for the first term, $\left\|\partial_{x}\left(u_{1} u_{2}\right)\right\|_{X_{s, b-1}}$, on the righthand side of the equality, as the proof for the second term is easier than that for the first term:

$$
\left\|\partial_{x}\left(u_{1} u_{2}\right)\right\|_{X_{s, b-1}} \leqslant C\left\|u_{1}\right\|_{\tilde{X}_{s, b^{\prime}}}\left\|u_{2}\right\|_{\tilde{X}_{s, b^{\prime}}} .
$$

By duality and the Plancherel identity, it suffices to show that

$$
\begin{aligned}
\Upsilon & =\int_{\star}\langle\xi\rangle^{s}|\xi| \frac{\bar{f}(\tau, \xi)}{\langle\sigma\rangle^{1-b}} \mathcal{F} u_{1}\left(\tau_{1}, \xi_{1}\right) \mathcal{F} u_{2}\left(\tau_{2}, \xi_{2}\right) \mathrm{d} \delta \\
& =\int_{\star} \frac{\langle\xi\rangle^{s}|\xi|}{\langle\sigma\rangle^{1-b} \prod_{j=1}^{2}\left\langle\xi_{j}\right\rangle^{s}\left\langle\sigma_{j}\right\rangle^{\prime}} \bar{f}(\tau, \xi) f_{1}\left(\tau_{1}, \xi_{1}\right) f_{2}\left(\tau_{2}, \xi_{2}\right) \mathrm{d} \delta \\
& \leqslant\left\|\frac{\langle\xi\rangle^{s}|\xi|}{\langle\sigma\rangle^{1-b} \prod_{j=1}^{2}\left\langle\xi_{j}\right\rangle^{s}\left\langle\sigma_{j}\right\rangle^{b^{\prime}}}\right\|_{[3 ; \mathbb{R} \times \mathbb{R}]}\|f\|_{L_{2}} \prod_{j=1}^{2}\left(\left\|f_{j}\right\|_{L_{2}}+\left\||\xi|^{-1} f_{j}\right\|_{L_{2}}\right),
\end{aligned}
$$

for $\bar{f} \in L_{2}, \bar{f} \geqslant 0$, where

$$
f_{j}=\left\langle\xi_{j}\right\rangle^{s}\left\langle\sigma_{j}\right\rangle^{b^{\prime}} \hat{u_{j}}, \quad j=1,2
$$

$\xi=\xi_{1}+\xi_{2}, \tau=\tau_{1}+\tau_{2}$.

One easily obtains that

$$
\left\|f_{j}\right\|_{L^{2}}+\left\||\xi|^{-1} f_{j}\right\|_{L_{2}}=\left\|u_{j}\right\|_{\tilde{X}_{s, b^{\prime}}} .
$$

Theorem 4.1 holds only if the following holds:

$$
\left\|\frac{\langle\xi\rangle^{s}|\xi|}{\langle\sigma\rangle^{1-b} \prod_{j=1}^{2}\left\langle\xi_{j}\right\rangle^{s}\left\langle\sigma_{j}\right\rangle^{b^{\prime}}}\right\|_{[3 ; \mathbb{R} \times \mathbb{R}]} \leqslant C .
$$


Let

$$
\mathcal{F} F_{\rho}^{j}(\xi, \tau)=\frac{f_{j}(\xi, \tau)}{\left(1+\left|\tau+\beta \xi^{3}+(\gamma / \xi)\right|\right)^{\rho}}, \quad j=1,2 .
$$

In order to bound the integral $\Upsilon$, we split the domain of integration into several pieces.

We consider the most interesting case $s \leqslant 0$. Let $r=-s$. By symmetry, it suffices to estimate the integral in the domain $\left|\xi_{1}\right| \leqslant\left|\xi_{2}\right|$.

Situation I. Assume that $|\xi| \leqslant 4 a$.

Case 1. If $\left|\xi_{1}\right| \leqslant 2 a$, then we have $\left|\xi_{2}\right| \leqslant\left|\xi-\xi_{1}\right| \leqslant 6 a$. Consequently, the integral $\Upsilon$ restricted to this domain is bounded by

$$
\begin{aligned}
\int_{\star} \frac{|\xi| \bar{f}(\tau, \xi)}{\langle\xi\rangle^{r}\langle\sigma\rangle^{1-b}} \frac{\left\langle\xi_{1}\right\rangle^{r} f_{1}\left(\tau_{1}, \xi_{1}\right)}{\left\langle\sigma_{1}\right\rangle^{b^{\prime}}} \frac{\left\langle\xi_{2}\right\rangle^{r} f_{2}\left(\tau_{2}, \xi_{2}\right)}{\left\langle\sigma_{2}\right\rangle^{b^{\prime}}} \mathrm{d} \delta & \leqslant C \int_{\star} \frac{\bar{f}(\tau, \xi)}{\langle\sigma\rangle^{1-b}} \frac{f_{1}\left(\tau_{1}, \xi_{1}\right)}{\left\langle\sigma_{1}\right\rangle^{b^{\prime}}} \frac{f_{2}\left(\tau_{2}, \xi_{2}\right)}{\left\langle\sigma_{2}\right\rangle^{b^{\prime}}} \mathrm{d} \delta \\
& \leqslant C \int \bar{F}_{1-b} F_{b^{\prime}}^{1} F_{b^{\prime}}^{2}(x, t) \mathrm{d} x \mathrm{~d} t \\
& \leqslant C\left\|F_{1-b}\right\|_{L_{x}^{2} L_{t}^{2}}\left\|F_{b^{\prime}}^{1}\right\|_{L_{x}^{4} L_{t}^{4}}\left\|F_{b^{\prime}}^{2}\right\|_{L_{x}^{4} L_{t}^{4}} \\
& \leqslant C\|f\|_{L_{\xi}^{2} L_{\tau}^{2}}\left\|f_{1}\right\|_{L_{\xi}^{2} L_{\tau}^{2}}\left\|f_{2}\right\|_{L_{\xi}^{2} L_{\tau}^{2}},
\end{aligned}
$$

which follows by Lemmas 2.7 and 2.8 .

Case 2. If $2 a \leqslant\left|\xi_{1}\right| \leqslant\left|\xi_{2}\right|$, then, for $r \leqslant \frac{1}{8}$, we have

$$
\begin{aligned}
\int_{\star} \frac{|\xi| \bar{f}(\tau, \xi)}{\langle\xi\rangle^{r}\langle\sigma\rangle^{1-b}} \frac{\left\langle\xi_{1}\right\rangle^{r} f_{1}\left(\tau_{1}, \xi_{1}\right)}{\left\langle\sigma_{1}\right\rangle^{b^{\prime}}} \frac{\left\langle\xi_{2}\right\rangle^{r} f_{2}\left(\tau_{2}, \xi_{2}\right)}{\left\langle\sigma_{2}\right\rangle^{b^{\prime}}} \mathrm{d} \delta \\
\quad \leqslant C \int_{\star} \frac{\bar{f}(\tau, \xi)}{\langle\sigma\rangle^{1-b}} \frac{\left\langle\xi_{1}\right\rangle^{r} \chi_{\left|\xi_{1}\right| \geqslant 2 a} f_{1}\left(\tau_{1}, \xi_{1}\right)}{\left\langle\sigma_{1}\right\rangle^{b^{\prime}}} \frac{\left\langle\xi_{2}\right\rangle^{r} \chi_{\left|\xi_{2}\right| \geqslant 2 a} f_{2}\left(\tau_{2}, \xi_{2}\right)}{\left\langle\sigma_{2}\right\rangle^{b^{\prime}}} \mathrm{d} \delta \\
\quad \leqslant C \int \bar{F}_{1-b} \cdot D_{x}^{1 / 8} P^{2 a} F_{b^{\prime}}^{1} \cdot D_{x}^{1 / 8} P^{2 a} F_{b^{\prime}}^{2}(x, t) \mathrm{d} x \mathrm{~d} t \\
\leqslant C\left\|F_{1-b}\right\|_{L_{x}^{2} L_{t}^{2}}\left\|D_{x}^{1 / 8} P^{2 a} F_{b^{\prime}}^{1}\right\|_{L_{x}^{4} L_{t}^{4}}\left\|D_{x}^{1 / 8} P^{2 a} F_{b^{\prime}}^{2}\right\|_{L_{x}^{4} L_{t}^{4}} \\
\leqslant C\|f\|_{L_{\xi}^{2} L_{\tau}^{2}}\left\|f_{1}\right\|_{L_{\xi}^{2} L_{\tau}^{2}}\left\|f_{2}\right\|_{L_{\xi}^{2} L_{\tau}^{2}},
\end{aligned}
$$

which follows by Lemmas 2.5 and 2.8 .

Situation II. Assume that $|\xi| \geqslant 4 a$.

Case 1. If $\left|\xi_{1}\right| \leqslant 2 a$, then $\left|\xi_{2}\right| \geqslant 2 a$ and $|\xi| \sim\left|\xi_{2}\right|$.

Subcase 1 . If $1 / 10 a \leqslant\left|\xi_{1}\right| \leqslant 2 a$, then

$$
\begin{gathered}
\int_{\star} \frac{|\xi| \bar{f}(\tau, \xi)}{\langle\xi\rangle^{r}\langle\sigma\rangle^{1-b}} \frac{\left\langle\xi_{1}\right\rangle^{r} f_{1}\left(\tau_{1}, \xi_{1}\right)}{\left\langle\sigma_{1}\right\rangle^{b^{\prime}}} \frac{\left\langle\xi_{2}\right\rangle^{r} f_{2}\left(\tau_{2}, \xi_{2}\right)}{\left\langle\sigma_{2}\right\rangle^{b^{\prime}}} \mathrm{d} \delta \\
\leqslant C \int_{\star} \frac{\bar{f}(\tau, \xi)}{\langle\sigma\rangle^{1-b}} \frac{\chi_{1 / 10 a \leqslant\left|\xi_{1}\right| \leqslant 2 a} f_{1}\left(\tau_{1}, \xi_{1}\right)}{\left\langle\sigma_{1}\right\rangle^{b^{\prime}}} \frac{\left|\xi_{2}\right| \chi_{\left|\xi_{2}\right| \geqslant 2 a} f_{2}\left(\tau_{2}, \xi_{2}\right)}{\left\langle\sigma_{2}\right\rangle^{b^{\prime}}} \mathrm{d} \delta \\
\leqslant C \int \bar{F}_{1-b} \cdot P_{(1 / 10 a, 2 a)} F_{b^{\prime}}^{1} \cdot D_{x} P^{2 a} F_{b^{\prime}}^{2}(x, t) \mathrm{d} x \mathrm{~d} t
\end{gathered}
$$




$$
\begin{aligned}
& \leqslant C\left\|F_{1-b}\right\|_{L_{x}^{2} L_{t}^{2}}\left\|P_{(1 / 10 a, 2 a)} F_{b^{\prime}}^{1}\right\|_{L_{x}^{2} L_{t}^{\infty}}\left\|D_{x} P^{2 a} F_{b^{\prime}}^{2}\right\|_{L_{x}^{\infty} L_{t}^{2}} \\
& \leqslant C\|f\|_{L_{\xi}^{2} L_{\tau}^{2}}\left\|f_{1}\right\|_{L_{\xi}^{2} L_{\tau}^{2}}\left\|f_{2}\right\|_{L_{\xi}^{2} L_{\tau}^{2}},
\end{aligned}
$$

which follows by Lemmas 2.4, 2.6 and 2.8 .

Subcase 2 . If $\left|\xi_{1}\right| \leqslant 1 / 10 a$, then we split the domain of integration into two pieces.

(i) If $\left|\xi_{1} \cdot \xi_{2}\right| \leqslant 4 a^{2}$, then the integral $\Upsilon$ is bounded by

$$
\begin{aligned}
& C \int_{\star} \frac{\bar{f}(\tau, \xi)}{\langle\sigma\rangle^{1-b}} \frac{f_{1}\left(\tau_{1}, \xi_{1}\right)}{\left\langle\sigma_{1}\right\rangle^{b^{\prime}}} \frac{\left|\xi_{2}\right| \chi_{\left|\xi_{2}\right| \geqslant 2 a} f_{2}\left(\tau_{2}, \xi_{2}\right)}{\left\langle\sigma_{2}\right\rangle^{b^{\prime}}} \mathrm{d} \delta \\
& \quad \leqslant C \int_{\star} \frac{\bar{f}(\tau, \xi)}{\langle\sigma\rangle^{1-b}} \frac{f_{1}\left(\tau_{1}, \xi_{1}\right)}{\left\langle\sigma_{1}\right\rangle^{b^{\prime}}\left|\xi_{1}\right|} \frac{f_{2}\left(\tau_{2}, \xi_{2}\right)}{\left\langle\sigma_{2}\right\rangle^{b^{\prime}}} \mathrm{d} \delta \\
& \leqslant C \int \bar{F}_{1-b} \cdot D_{x}^{-1} F_{b^{\prime}}^{1} \cdot F_{b^{\prime}}^{2}(x, t) \mathrm{d} x \mathrm{~d} t \\
& \leqslant C\left\|F_{1-b}\right\|_{L_{x}^{2} L_{t}^{2}}\left\|D_{x}^{-1} F_{b^{\prime}}^{1}\right\|_{L_{x}^{4} L_{t}^{4}}\left\|D_{x} P^{2 a} F_{b^{\prime}}^{2}\right\|_{L_{x}^{4} L_{t}^{4}} \\
& \leqslant C\|f\|_{L_{\xi}^{2} L_{\tau}^{2}}\left\|\left.\xi\right|^{-1} f_{1}\right\|_{L_{\xi}^{2} L_{\tau}^{2}}\left\|f_{2}\right\|_{L_{\xi}^{2} L_{\tau}^{2}},
\end{aligned}
$$

which follows by Lemmas 2.7 and 2.8.

(ii) If $\left|\xi_{1} \cdot \xi_{2}\right| \geqslant 4 a^{2}$, then from the identities (1.8), it follows that

$$
\max \left(|\sigma|,\left|\sigma_{1}\right|, \mid \sigma_{2}\right)|\geqslant C| \xi \xi_{1} \xi_{2} \mid .
$$

For simplicity, we assume that

$$
|\sigma| \geqslant C\left|\xi \xi_{1} \xi_{2}\right|
$$

Then, for $2(1-b) \leqslant \frac{7}{8}$, the integral $\Upsilon$ is bounded by

$$
\begin{aligned}
& \int_{\star} \frac{|\xi| \bar{f}(\tau, \xi)}{\langle\xi\rangle^{r}\left|\xi \xi_{1} \xi_{2}\right|^{1-b}} \frac{\left\langle\xi_{1}\right\rangle^{r} f_{1}\left(\tau_{1}, \xi_{1}\right)}{\left\langle\sigma_{1}\right\rangle^{b^{\prime}}} \frac{\left\langle\xi_{2}\right\rangle^{r} f_{2}\left(\tau_{2}, \xi_{2}\right)}{\left\langle\sigma_{2}\right\rangle^{b^{\prime}}} \mathrm{d} \delta \\
& \quad \leqslant C \int_{\star} \bar{f}(\tau, \xi) \frac{f_{1}\left(\tau_{1}, \xi_{1}\right)}{\left\langle\sigma_{1}\right\rangle^{b^{\prime}}\left|\xi_{1}\right|} \frac{\left|\xi_{2}\right|^{1 / 8} \chi_{\left|\xi_{2}\right| \geqslant 2 a} f_{2}\left(\tau_{2}, \xi_{2}\right)}{\left\langle\sigma_{2}\right\rangle^{b^{\prime}}} \mathrm{d} \delta \\
& \leqslant C \int \bar{F}_{0} \cdot D_{x}^{-1} F_{b^{\prime}}^{1} \cdot D_{x}^{1 / 8} P^{2 a} F_{b^{\prime}}^{2}(x, t) \mathrm{d} x \mathrm{~d} t \\
& \leqslant C\left\|F_{0}\right\|_{L_{x}^{2} L_{t}^{2}}\left\|D_{x}^{-1} F_{b^{\prime}}^{1}\right\|_{L_{x}^{4} L_{t}^{4}}\left\|D_{x}^{1 / 8} P^{2 a} F_{b^{\prime}}^{2}\right\|_{L_{x}^{4} L_{t}^{4}} \\
& \leqslant C\|f\|_{L_{\xi}^{2} L_{\tau}^{2}}\left\|\left.\xi\right|^{-1 \mid} f_{1}\right\|_{L_{\xi}^{2} L_{\tau}^{2}}\left\|f_{2}\right\|_{L_{\xi}^{2} L_{\tau}^{2}},
\end{aligned}
$$

which follows by Lemmas 2.4, 2.6 and 2.8 .

Case 2. If $2 a \leqslant\left|\xi_{1}\right| \leqslant\left|\xi_{2}\right|$, then from the identities (1.8), it follows that, if $|\xi| \geqslant a$, $\left|\xi_{1}\right| \geqslant a$ and $\left|\xi_{2}\right| \geqslant a$, then

$$
\max \left(|\sigma|,\left|\sigma_{1}\right|, \mid \sigma_{2}\right)|\geqslant C| \xi \xi_{1} \xi_{2} \mid .
$$

This implies that one of the following cases always occurs: 
(a) $|\sigma| \geqslant C\left|\xi \xi_{1} \xi_{2}\right|$,

(b) $\left|\sigma_{1}\right| \geqslant C\left|\xi \xi_{1} \xi_{2}\right|$,

(c) $\left|\sigma_{2}\right| \geqslant C\left|\xi \xi_{1} \xi_{2}\right|$.

In this domain (Case 2), the integral $\Upsilon$ is bounded by

$$
\int_{\star} \frac{|\xi|^{1-r} \chi_{|\xi| \geqslant 4 a} \bar{f}(\tau, \xi)}{\langle\sigma\rangle^{1-b}} \frac{\left\langle\xi_{1}\right\rangle^{r} \chi_{\left|\xi_{1}\right| \geqslant 2 a} f_{1}\left(\tau_{1}, \xi_{1}\right)}{\left\langle\sigma_{1}\right\rangle^{b^{\prime}}} \frac{\left\langle\xi_{2}\right\rangle^{r} \chi_{\left|\xi_{2}\right| \geqslant 2 a} f_{2}\left(\tau_{2}, \xi_{2}\right)}{\left\langle\sigma_{2}\right\rangle^{b^{\prime}}} \mathrm{d} \delta .
$$

We consider the three cases (a), (b) and (c) separately.

If (a) holds, for $r+b-1 \leqslant \frac{1}{8}$ and $r \geqslant b$, we obtain

$$
\begin{aligned}
& \int_{\star} \frac{|\xi|^{1-r} \chi_{|\xi| \geqslant 4 a} \bar{f}(\tau, \xi)}{\left(\left|\xi_{1}\right|\left|\xi_{2}\right||\xi|\right)^{1-b}} \frac{\left\langle\xi_{1}\right\rangle^{r} \chi_{\left|\xi_{1}\right| \geqslant 2 a} f_{1}\left(\tau_{1}, \xi_{1}\right)}{\left\langle\sigma_{1}\right\rangle^{b^{\prime}}} \frac{\left\langle\xi_{2}\right\rangle^{r} \chi_{\left|\xi_{2}\right| \geqslant 2 a} f_{2}\left(\tau_{2}, \xi_{2}\right)}{\left\langle\sigma_{2}\right\rangle^{b^{\prime}}} \mathrm{d} \delta \\
& \quad \leqslant C \int_{\star}|\xi|^{b-r} \chi_{|\xi| \geqslant 4 a} \bar{f}(\tau, \xi) \frac{\left|\xi_{1}\right|^{r+b-1} \chi_{\left|\xi_{1}\right| \geqslant 2 a} f_{1}\left(\tau_{1}, \xi_{1}\right)}{\left\langle\sigma_{1}\right\rangle^{b^{\prime}}} \frac{\left|\xi_{2}\right|^{r+b-1} \chi_{\left|\xi_{2}\right| \geqslant 2 a} f_{2}\left(\tau_{2}, \xi_{2}\right)}{\left\langle\sigma_{2}\right\rangle^{b^{\prime}}} \mathrm{d} \delta \\
& \quad \leqslant C \int \bar{F}_{0} \cdot D_{x}^{1 / 8} P^{2 a} F_{b^{\prime}}^{1} \cdot D_{x}^{1 / 8} P^{2 a} F_{b^{\prime}}^{2}(x, t) \mathrm{d} x \mathrm{~d} t \\
& \quad \leqslant C\left\|F_{0}\right\|_{L_{x}^{2} L_{t}^{2}}\left\|D_{x}^{1 / 8} P^{2 a} F_{b^{\prime}}^{1}\right\|_{L_{x}^{4} L_{t}^{4}}\left\|D_{x}^{1 / 8} P^{2 a} F_{b^{\prime}}^{2}\right\|_{L_{x}^{4} L_{t}^{4}} \\
& \quad \leqslant C\|f\|_{L_{\xi}^{2} L_{\tau}^{2}}\left\|f_{1}\right\|_{L_{\xi}^{2} L_{\tau}^{2}}\left\|f_{2}\right\|_{L_{\xi}^{2} L_{\tau}^{2}},
\end{aligned}
$$

which follows by Lemmas 2.5 and 2.8 .

This means that, if $r+b-1 \leqslant \frac{1}{8}, r \geqslant b$, then we have

$$
\left\|\frac{|\xi|\left\langle\xi_{1}\right\rangle^{r}\left\langle\xi_{2}\right\rangle^{r}}{\langle\sigma\rangle^{1-b}\langle\xi\rangle^{r}\left\langle\sigma_{1}\right\rangle^{b^{\prime}}\left\langle\sigma_{2}\right\rangle^{b^{\prime}}}\right\|_{[3 ; \mathbb{R} \times \mathbb{R}]} \leqslant C .
$$

However, if $r \leqslant \frac{1}{8}$, then by Lemma 2.9 we have

$$
\left\|\frac{|\xi|\left\langle\xi_{1}\right\rangle^{r}\left\langle\xi_{2}\right\rangle^{r}}{\langle\sigma\rangle^{1-b}\langle\xi\rangle^{r}\left\langle\sigma_{1}\right\rangle^{b^{\prime}}\left\langle\sigma_{2}\right\rangle^{b^{\prime}}}\right\|_{[3 ; \mathbb{R} \times \mathbb{R}]} \leqslant C .
$$

In fact, if $r_{1} \leqslant r_{2}$, by $\xi=\xi_{1}+\xi_{2}$ we obtain

$$
\frac{|\xi|\left\langle\xi_{1}\right\rangle^{r_{1}}\left\langle\xi_{2}\right\rangle^{r_{1}}}{\langle\sigma\rangle^{1-b}\langle\xi\rangle^{r_{1}}\left\langle\sigma_{1}\right\rangle^{b^{\prime}}\left\langle\sigma_{2}\right\rangle^{b^{\prime}}} \leqslant C \frac{|\xi|\left\langle\xi_{1}\right\rangle^{r_{2}}\left\langle\xi_{2}\right\rangle^{r_{2}}}{\langle\sigma\rangle^{1-b}\langle\xi\rangle^{r_{2}}\left\langle\sigma_{1}\right\rangle^{b^{\prime}}\left\langle\sigma_{2}\right\rangle^{b^{\prime}}} .
$$

If (b) holds, for $r+b^{\prime} \geqslant 1, r-b^{\prime} \leqslant \frac{1}{16}$, then we get

$$
\begin{aligned}
& \int_{\star} \frac{|\xi|^{1-r} \chi_{|\xi| \geqslant 4 a} \bar{f}(\tau, \xi)}{\left\langle\sigma_{1}\right\rangle 1-b} \frac{\left|\xi_{1}\right|^{r} \chi_{\left|\xi_{1}\right| \geqslant 2 a} f_{1}\left(\tau_{1}, \xi_{1}\right)}{\left(\left|\xi_{1}\right|\left|\xi_{2}\right||\xi|\right)^{b^{\prime}}} \frac{\left\langle\xi_{2}\right\rangle^{r} \chi_{\left|\xi_{2}\right| \geqslant 2 a} f_{2}\left(\tau_{2}, \xi_{2}\right)}{\left\langle\sigma_{2}\right\rangle^{b^{\prime}}} \mathrm{d} \delta \\
& \quad \leqslant C \int_{\star} \frac{|\xi|^{1-b^{\prime}-r} \chi_{|\xi| \geqslant 4 a} \bar{f}(\tau, \xi)}{\left\langle\sigma_{1}\right\rangle^{1-b}} \chi_{\left|\xi_{1}\right| \geqslant 2 a} f_{1}\left(\tau_{1}, \xi_{1}\right) \frac{\left|\xi_{2}\right|^{2\left(r-b^{\prime}\right)} \chi_{\left|\xi_{2}\right| \geqslant 2 a} f_{2}\left(\tau_{2}, \xi_{2}\right)}{\left\langle\sigma_{2}\right\rangle^{b^{\prime}}} \mathrm{d} \delta \\
& \quad \leqslant C \int \bar{F}_{1-b} \cdot F_{0}^{1} \cdot D_{x}^{1 / 8} P^{2 a} F_{b^{\prime}}^{2}(x, t) \mathrm{d} x \mathrm{~d} t
\end{aligned}
$$




$$
\begin{aligned}
& \leqslant C\left\|F_{1-b}\right\|_{L_{x}^{4} L_{t}^{4}}\left\|F_{0}^{1}\right\|_{L_{x}^{2} L_{t}^{2}}\left\|D_{x}^{1 / 8} P^{2 a} F_{b^{\prime}}^{2}\right\|_{L_{x}^{4} L_{t}^{4}} \\
& \leqslant C\|f\|_{L_{\xi}^{2} L_{\tau}^{2}}\left\|f_{1}\right\|_{L_{\xi}^{2} L_{\tau}^{2}}\left\|f_{2}\right\|_{L_{\xi}^{2} L_{\tau}^{2}},
\end{aligned}
$$

which follows by Lemmas 2.5, 2.7 and 2.8.

In a similar way to the treatment of (a) above, if $r \leqslant \frac{1}{8}$, by Lemma 2.9, we get

$$
\left\|\frac{|\xi|\left\langle\xi_{1}\right\rangle^{r}\left\langle\xi_{2}\right\rangle^{r}}{\langle\sigma\rangle^{1-b}\langle\xi\rangle^{r}\left\langle\sigma_{1}\right\rangle^{b^{\prime}}\left\langle\sigma_{2}\right\rangle^{b^{\prime}}}\right\|_{[3 ; \mathbb{R} \times \mathbb{R}]} \leqslant C .
$$

If (c) holds, the argument is similar to case (b). This completes the proof of Theorem 4.1 .

\section{The proof of Theorem 1.2}

Now we turn to the proof of Theorem 1.2.

For $u_{0} \in \tilde{H}^{s}\left(s \geqslant-\frac{1}{8}\right)$, we define the operator

$$
\Phi(u)=\psi_{1}(t) S(t) u_{0}+\psi_{1}(t) \int_{0}^{t} S\left(t-t^{\prime}\right) \psi_{\delta}\left(t^{\prime}\right) \partial_{x}\left(u^{2}\right)\left(t^{\prime}\right) \mathrm{d} t^{\prime}
$$

and the set

$$
\mathcal{B}=\left\{u \in \tilde{X}_{s, b}:\|u\|_{\tilde{X}_{s, b}} \leqslant 2 C\left\|u_{0}\right\|_{\tilde{H}^{s}}\right\} .
$$

In order to show that $\Phi$ is a contraction mapping on $\mathcal{B}$, we first prove

$$
\Phi(\mathcal{B}) \subset \mathcal{B}
$$

By Theorem 4.1 and Lemmas 3.1-3.3 for $\frac{1}{2}<b<b^{\prime}<1$, we have the next chain of inequalities:

$$
\|\Phi(u)\|_{\tilde{X}_{s, b}} \leqslant C\left\|u_{0}\right\|_{\tilde{H}^{s}}+C \delta^{b^{\prime}-b}\|u\|_{\tilde{X}_{s, b}}^{2} \leqslant C\left\|u_{0}\right\|_{\tilde{H}^{s}}+C \delta^{b^{\prime}-b}\left\|u_{0}\right\|_{\tilde{H}^{s}}\|u\|_{\tilde{X}_{s, b}} .
$$

Therefore, if we fix $\delta$ such that $C \delta^{b^{\prime}-b}\left\|u_{0}\right\|_{\tilde{H}^{s}} \leqslant \frac{1}{2}$, then we have

$$
\Phi(\mathcal{B}) \subset \mathcal{B} .
$$

For $u, v \in \mathcal{B}$, reasoning in an analogous way to above, it follows that

$$
\|\Phi(u)-\Phi(v)\|_{\tilde{X}_{s, b}} \leqslant C \delta^{b^{\prime}-b}\left(\|u\|_{\tilde{X}_{s, b}}+\|v\|_{\tilde{X}_{s, b}}\right)\|u-v\|_{\tilde{X}_{s, b}} \leqslant \frac{1}{2}\|u-v\|_{\tilde{X}_{s, b}} .
$$

Therefore, $\Phi$ is a contraction mapping on $\mathcal{B}$. There exists a unique fixed point which solves the Cauchy problem (1.1), (1.2) for $T<\frac{1}{2} \delta$.

Acknowledgements. This work was supported by the China Postdoctoral Science Foundation and the National Natural Science Fund. 


\section{References}

1. E. S. Benilov, On the surface waves in a shallow channel with an uneven bottom, Stud. Appl. Math. 87 (1992), 1-14.

2. J. BerGH AND J. LÖFSTRÖM, Interpolation spaces (Springer, 1976).

3. J. Bourgain, Fourier transform restriction phenomena for certain lattice subsets and applications to nonlinear evolution equations, Part I, Schrödinger equations, Geom. Funct. Analysis 3 (1993), 107-156.

4. J. Bourgain, Fourier restriction phenomena for certain lattice subsets and applications to nonlinear evolution equations, Part II, The KdV equation, Geom. Funct. Analysis 2 (1993), 209-262.

5. V. N. Galkin and Yu. A. Stepanyants, On the existence of stationary solitary waves in a rotating fliud, J. Appl. Math. Mech. 55 (1991), 939-943.

6. O. A. Gilman, R. Grimshaw and Yu. A. Stepanyants, Approximate and numerical solutions of the stationary Ostrovsky equation, Stud. Appl. Math. 95 (1995), 115-126.

7. C. E. Kenig, G. Ponce And L. VeGA, Oscillatory integrals and regularity of dispersive equations, Indiana Univ. Math. J. 40 (1991), 33-69.

8. C. E. Kenig, G. Ponce And L. Vega, The Cauchy problem for the Korteweg-de Vries equation in Sobolev spaces of negative indices, Duke Math. J. 71 (1993), 1-21.

9. C. E. Kenig, G. Ponce And L. Vega, A bilinear estimate with applications to the KdV equation, J. Am. Math. Soc. 9 (1996), 573-603.

10. L. A. Ostrovsky, Nonlinear internal waves in a rotating ocean, Okeanologia (2) 18 (1978), 181-191.

11. E. M. STEIN, Harmonic analysis, real-variable methods, orthogonality and oscillatroy integrals (Princeton University Press, 1993).

12. T. TAO, Multilinear weighted convolution of $L^{2}$ functions, and applications to nonlinear dispersive equations, Am. J. Math. 123 (2001), 839-908.

13. V. Varlamov and Y. LiU, Cauchy problem for the Ostrovsky equation, Discrete Contin. Dynam. Syst. 10 (2004), 731-753. 